\title{
The Application of Next Generation Sequencing in Cancer Precision Diagnosis and Target Therapy Selection
}

\author{
Tingting Lin ${ }^{1}$ and Peng Lyu ${ }^{2 *}$ \\ ${ }^{1}$ Department of Pharmacy, Zhongshan Hospital FuDan University, China \\ ${ }^{2}$ College of Biological Science and Technology, Fuzhou University, China \\ *Corresponding author: Peng Lyu, College of Biological Science and Technology, Fuzhou University, Fujian, China
}

\section{ARTICLE INFO}

Received: 慧 July 22, 2019

Published: 幽 July 30, 2019
ABSTRACT

Citation: Tingting Lin, Peng Lyu. The Application of Next Generation Sequencing in Cancer Precision Diagnosis and Target Therapy Selection. Biomed J Sci \& Tech Res 20(1)-2019. BJSTR. MS.ID.003405.

\section{Introduction}

Cancer comprises more than 100 distinct human malignancies and is highly heterogeneous in its genetic and molecular aspects [1]. Researches using cytogenetic techniques and high-throughput genome-wide approaches have made significant contributions for cancer treatments that involve the specific target therapies [2]. Antibody-based therapy for cancer has been established more than 15 years and is now one of the most successful and important strategy for treating patients with haematological malignancies and solid tumours condition [3]. The development of successful and applicable therapeutic antibodies requires a thorough understanding of cancer serology, protein-engineering techniques, mechanisms of action and resistance, and the crosslink between the immune system and cancer cells. It is not surprising that recent and emerging R\&D pharmaceutical and biomolecular diagnostic industries have shifted to signalling transduction-inhibitor drugs, such as, target immune checkpoint drugs and antibody therapies. The monoclonal antibody products dramatically climbing in the global market from 2010 , which was nearly $\$ 75$ billion annually [4]

This steady market-based sales growth of the current approved monoclonal antibody products, along with over 300 monoclonal antibody product candidates, which are in their development phase is clearly projecting a time-series forecast and predicted to drive the overall sales of all biopharmaceutical products. 4 Unlike chemotherapies, the monoclonal antibody products exclusively rely on the specific gene alteration of cancer patients. The traditional cancer diagnosis methods use diagnostic imaging and biopsy is still equally essential. However, there is a demand for a more efficient and comprehensive approach to provide precision diagnosis based on the genetic diversity and genomic alteration of cancer patients. This is simply because each cancer patient has distinct genome and health condition. Next Generation Sequencing (NGS) has many advantages, such as, the capability to fully sequence every type of mutations in a large number of genes (hundreds to thousands) without sacrificing sensitivity and speed in a single test at a relatively low cost compared to other sequencing modalities. Till date, the development of NGS platforms, like RNA sequencing (RNA-seq), and whole-genome sequencing (WGS), have greatly expediated the cancer research. Particularly, RNA-Seq is expected to play a key role to promote the tumour diagnostic and therapeutic biomarkers/targets discovery and precision medicine due to its comprehensive, high effort and accurate detection of transcriptome alteration of cancer patients.

\section{Diagnosis Application of High-throughput Method}

Historically, the quantitative reverse transcription PCR (qRTPCR) was used as the 'gold standard' method in gene transcriptional detection and predominate in the clinical aspect. The benefits of qPCR include fast, accurate, sensitive and low-sample requirement allows the extensive application of qPCR in both clinical and laboratory researches. However, there are multiple obstacles identified for qPCR in the precision diagnosis, which is essential 
for the target therapy selection. With the increase of the number of target therapies, hundreds to thousands of genes need to be detected to select the suitable target therapies. Thus, relatively few markers can be measured in every single assay greatly enhance the time cost for qPCR. Amid the development of NGS technology, lesser consumable, time and cost are now applied into clinical laboratories for diagnostic testing for multicancer patients [5]. In the past decade, NGS-based genomic study is widely used in cancer diagnosis. The NGS-based diagnosis strategy showed potency for ovarian cancer [6], breast cancer [7] and thyroid cancer [8]. In the era of NGS, RNA-seq is the most frequently used methods for cancer diagnosis and research. This in-depth and rapid transcriptome detection provides unprecedented flexibility, sensitivity and accuracy of gene expression measurements. Hundreds of therapeutic target genes can be detected in a single assay, which allowed the precision genomic alteration detection. Based on the RNA-seq strategy, more accurate diagnosis and treatment can be made to improve the prognostic result for different cancer patients. For instance, the karyotyping and qRT-PCR measurement of BCRABL1 was suggested to be used for the diagnosis of chronic myeloid leukaemia (CML) [9]. Recently, the development of NGS technology application has promised to further explore into the poor prognostic CML patients [9]. Translocation and RUNX1-RUNX1T1 fusion, also known as AML1-ETO, has been recognised as a diagnostic genome alteration of acute myeloid leukaemia (AML) [10]. TMPRSS2-ERG fusion was found associated with prostate cancer [11]. Therefore, it is obvious that the adoption of RNA-seq has high potential and promising in the precision and early diagnosis, and treatment for cancer patients.

\section{Assessment of Target Therapy}

Indeed, personalised diagnostic is capable to improve the selection of target therapies for heterogeneity cancers. Despite that, emerging development of NGS technology has been applied beyond cancer diagnostic research. This is seen in several target therapies approved by the FDA, such as EGFR-TKIs, kinase inhibitors, PD-1/ PD-L1 inhibitor and ALK inhibitors, have already achieved great success in different cancer types. However, compared to the traditional chemotherapies, target therapies have limited anticancer spectrum. Certain genomic alteration of cancer cells resulted in achieved target therapy resistance, including EGFR T790M [12]. Currently, alteration of the alternatively spliced androgen receptor variant 7 (AR-V7) was found to be linked with castration-resistant in prostate cancer [13]. EML4-ALK fusion was originally reported in non-small-cell lung cancers, [14] which is targetable by ALKspecific inhibitors such as crizotinib. The genomic alteration seems to be a double-edged sword in tumour progression. Some gene alteration provided the therapeutic targets for cancer treatment but others extremely accelerated tumour development and invasion. Thus, landscape genomic sequencing by using NGS may avoid the missing of tumour progression associated gene alterations. Besides, the continuous NGS data collection is now able to aid the discovery of specific transcripts and genomic alteration of tumor, which might be the potential cancer therapeutic targets.

The genomic alteration of cancer does not only happen in the tumourigenesis state. For instance, acquired resistance to EGFR-TKIs usually developed after a period of treatment with the gefitinib or erlotinib [15]. One of the hypotheses revealed that the induced acquired resistant is the point mutation of T790M of EGFR and c-MET amplification [16]. Therefore, more extensive evaluation of potential gene fusions and mutations might be helpful for the improvement of the prognosis of cancer patients. For example, the RNA-seq-based clinical detection was used for the evaluation of common gene fusions prognostic situation in associated with sarcomas $[17,18]$. Based on the RNA-seq results, the clinicians can capture the tumour promoting genomic alteration and fusion. Subsequently designed the precision treatment according to the genome of the patients. Based on comprehensive genome alteration of intratumour heterogeneity detected by using NGS, deeper insights into signalling pathway networks and development of next-generation drugs and biomarkers [19-21].

\section{Discovery of Novel Therapeutic Targets/Biomarkers}

Genetic biomarkers can be used for cancer diagnosis, prognosis, and target therapy selection. There are a variety of oncogene alterations, including BRCA1/2, Tp53, PTEN, CDH1, STK11, MLH1, MSH2, MSH6, PMS2, and PALB2, the lifetime risk rate of breast cancer is over $20 \%$ for the listed gene mutations [22]. Therefore, NGS-based gene panels have been developed to find new therapeutic and diagnostic targets for cancer treatment $[23,24]$. NGS-based diagnostic and target therapy selection has been greatly developed over the years, but, in the novel therapeutic targets/biomarkers' discovery aspect, invasively NGS which requires a biopsy sample is still not an ideal strategy. Non-invasive biofluids, such as, blood or serum, which contain abundant potential diagnostic and prognostic biomarkers such as RNA, circular RNA and proteins have been used in the research of cancer biomarker discovery [25].

Compared with protein biomarkers, RNA serves as the transmitters of hereditary information and regulator of transcription $[26,27]$. The alteration of RNA is able to provide dynamic insights into cellular states and regulatory processes of tumor. Circular RNA existed in plasma and/or serum allowed the development of non-invasive diagnosis and prognosis in the future $[28,29]$. Recently, with the conducted of worldwide collaboration projects such as The Cancer Genome Atlas (TCGA) and International Human Genome Project International Cancer Genome Consortium (ICGC), various potential RNA biomarkers have been successfully identified [30]. Multi gene expression panels such as PAM50, have been applied in the clinical diagnosis of breast cancer [31]. Other cell cycle progression, gene panel was used in the diagnosis of recurrence of prostate cancer.32 Non-coding RNA, including microRNAs (miRNAs) and long non-coding RNAs (lncRNA) are also considered to link with the tumour process and recurrence [33]. Moreover, miRNAs are poten- 
tial therapeutic targets in many cancer types.33 Furthermore, the comprehensive RNA alteration can be detected by RNA-seq-based NGS, which may support the discovery of therapeutic biomarkers/ targets [34].

\section{Prospect for the Next Frontier in Cancer Diagnosis}

With the rapid development of NGS technology and a fast pace declining of the costs-per-base sequenced over the past decade. The cost of deep genomic sequence drops drastically from to 3 billion dollars to a few thousand dollars [35]. According to the dramatically cost drop in sequencing service, the NGS is projected to participate in most of the cancer diagnostic and drug discovery aspects in near future [36]. WGS sequencing will perform the personalised 'precision medicine' [37,38], which aims to optimise the target therapy selection and prognosis for cancer patients $[39,40]$. In an interesting research, Friend and Ideker established a novel model based on several databases to visualise personalised healthcare setting [41]. Their model unravelled that precision diagnosis and treatment strategy can be actually made by clinicians.41 However, application NGS to the clinical setting is not easy, several challenges and disagreements have obstructed this application. One of the crucial issues is the complexity of tumour heterogeneity, comprehensive sequencing of tumours using NGS revealed hundreds of genomic alterations in each cancer [42-49].

There is still a long way to go before we can fully comprehend the biological significance of each of these mutational findings. Positively, researchers already achieved great successes in the aspect of target therapies against certain cell mutations. 49 Another obstacle is the data process after sequencing. The cost of NGS may be lower to $\$ 500 /$ genome or even $\$ 100 /$ genome after a few years. Experience analyser to process the dramatically large amount of sequencing data and revealed the biological signature in this data are still urgently required. The shortage of bioinformatician with core skills, including computer science, programming, biology, and statistics is a real bottleneck of the clinical application of NGS. The knowledge supply must catch up to be on par to serve the demand of advance technology to provide smooth and accurate interpretation, or, one of the ultimate prowess of this application will not reach its potential. Even though there are still many challenges and obstacle for the researcher and clinicians. The advent of NGS accelerated the development of precision cancer diagnosis and treatment. The detailed genome landscape detected by NGS also facilitated the discovery of novel therapeutic biomarkers/targets. In the near future, NGS has a huge opportunity to revolutionise the treatment and diagnosis of cancer.

\section{References}

1. Stratton MR, Campbell PJ, Futreal PA (2009) The cancer genome. Nature 458: 719.

2. Ke X, Shen L (2017) Molecular targeted therapy of cancer: The progress and future prospect. Frontiers in Laboratory Medicine 1(2): 69-75.

3. Scott AM, Wolchok JD, Old LJ (2012) Antibody therapy of cancer. Nature reviews cancer $12(4)$ : 278-287.
4. Ecker DM, Jones SD, Levine HL (2015) The therapeutic monoclonal antibody market. MAbs. 7(1): 9-14.

5. Klee EW, Hoppman Chaney NL, Ferber MJ (2011) Expanding DNA diagnostic panel testing: is better? Expert review of molecular diagnostics 11(7): 703-709.

6. Park JY, Kricka LJ, Clark P, Londin E, Fortina P (2014) Clinical genomics: when whole genome sequencing is like a whole-body CT scan. Clinical chemistry 60(11): 1390-1392.

7. Castéra L, Krieger S, Rousselin A, Legros A, Baumann JJ, et al. (2014) Next-generation sequencing for the diagnosis of hereditary breast and ovarian cancer using genomic capture targeting multiple candidate genes. European Journal of Human Genetics 22(11): 1305-1313.

8. Nikiforova MN, Wald AI, Roy S, Durso MB, Nikiforov YE (2013) Targeted next-generation sequencing panel (ThyroSeq) for detection of mutations in thyroid cancer. The Journal of Clinical Endocrinology \& Metabolism 98(11): E1852-E1860.

9. Baran Y, Saydam G (2012) Cumulative clinical experience from a decade of use: imatinib as first-line treatment of chronic myeloid leukemia. Journal of blood medicine 3: 139-150.

10. Vardiman JW, Thiele J, Arber DA, Brunning RD, Borowitz MJ, et al. (2009) The 2008 revision of the World Health Organization (WHO) classification of myeloid neoplasms and acute leukemia: rationale and important changes. Blood 114(5): 937-951.

11. Steurer S (2014) TMPRSS2-ERG fusions are strongly linked to young patient age in low-grade prostate cancer. European urology 66(6): 978981.

12. Zhou W (2009) Novel mutant-selective EGFR kinase inhibitors against EGFR T790M. Nature 462(7276): 1070-1074.

13. Antonarakis ES (2015) Androgen receptor splice variant 7 and efficacy of taxane chemotherapy in patients with metastatic castration-resistant prostate cancer. JAMA oncology 1(5): 582-591.

14. Tetsumoto S (2013) Echinoderm Microtubule-Associated ProteinLike 4 (EML4)-Anaplastic Lymphoma Kinase (ALK) Rearrangement in Congenital Pulmonary Airway Malformation. Clinical lung cancer 14(4): 457-460.

15. Lin Y, Wang X, Jin H (2014) EGFR-TKI resistance in NSCLC patients: mechanisms and strategies. American journal of cancer research 4(5): 411-435.

16. Hata A (2013) Rebiopsy of non-small cell lung cancer patients with acquired resistance to epidermal growth factor receptor-tyrosine kinase inhibitor: comparison between t790m mutation-positive and mutationnegative populations. Cancer 119(24): 4325-4332.

17. Doebele RC (2015) An oncogenic NTRK fusion in a soft tissue sarcoma patient with response to the tropomyosin-related kinase (TRK) inhibitor LOXO-101. US Patent Application No 5(10): 1049-1057.

18. Sonu RJ, Jonas BA, Dwyre DM, Gregg JP, Rashidi HH (2015) Optimal Molecular Methods in Detecting p190BCR-ABL Fusion Variants in Hematologic Malignancies: A Case Report and Review of the Literature. Case reports in hematology 2015

19. Gerlinger M (2012) Intratumor heterogeneity and branched evolution revealed by multiregion sequencing. New Engl J Med 366(10): 883-892.

20. Ideker T, Krogan NJ (2012) Differential network biology. Molecular systems biology 8: 565 .

21. Roukos DH (2012) Differential signaling transduction networks for clinical robustness. Expert Rev Proteomic 9(2): 111-114.

22. Grignol VP, Agnese DM (2016) Breast cancer genetics for the surgeon: an update on causes and testing options. Journal of the American College of Surgeons 222(5): 906-914.

23. Easton DF (2015) Gene-panel sequencing and the prediction of breastcancer risk. New Engl J Med 372: 2243-2257. 
24. Chong HK (2014) The validation and clinical implementation of BRCA plus: a comprehensive high-risk breast cancer diagnostic assay. PloS one 9(5): e97408

25. Mishra A, Verma M (2010) Cancer biomarkers: are we ready for the prime time? Cancers 2(1): 190-208.

26. Yang YCT (2015) CLIPdb: a CLIP-seq database for protein-RNA interactions. BMC genomics 16: 51.

27. Hu B, Yang YCT, Huang Y, Zhu Y, Lu ZJ (2016) POSTAR: a platform for exploring post-transcriptional regulation coordinated by RNA-binding proteins. Nucleic acids research 45: D104-D114.

28. Chen LL (2016) The biogenesis and emerging roles of circular RNAs. Nature reviews Molecular cell biology 17(4): 205-211.

29. Memczak S (2013) Circular RNAs are a large class of animal RNAs with regulatory potency. Nature 495: 333-338.

30. Xi X (2017) RNA biomarkers: frontier of precision medicine for cancer Non-coding RNA 3(1): 9.

31. Parker JS (2009) Supervised risk predictor of breast cancer based on intrinsic subtypes. J Clin Oncol 27(8): 1160-1167.

32. Cuzick J (2011) Prognostic value of an RNA expression signature derived from cell cycle proliferation genes in patients with prostate cancer: a retrospective study. The lancet oncology 12(3): 245-255.

33. Glavac D, Hrasovec S (2012) MicroRNAs as novel biomarkers in colorectal cancer. Frontiers in genetics 3: 180.

34. Yang W, Ying D, Lau YL (2009) In-depth cDNA library sequencing provides quantitative gene expression profiling in cancer biomarker discovery. Genomics, proteomics \& bioinformatics 7(1-2): 1-12.

35. Drmana R (2010) Human genome sequencing using unchained base reads on self-assembling DNA nanoarrays. Science 327: 78-81.

36. Service RF (2006) Gene sequencing. The race for the $\$ 1000$ genome. Science (New York, NY) 311: 1544-1546.

37. Kahvejian A, Quackenbush J, Thompson JF (2008) What would you do if you could sequence everything? Nature biotechnology 26: 1125-1133.

\section{ISSN: 2574-1241}

DOI: $10.26717 /$ BJSTR.2019.20.003405

Peng Lyu. Biomed J Sci \& Tech Res

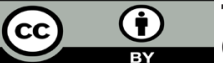

This work is licensed under Creative Commons Attribution 4.0 License

Submission Link: https://biomedres.us/submit-manuscript.php
38. Bogusk MS, Arnaout R, Hill C (2009) Customized care 2020: how medical sequencing and network biology will enable personalized medicine. F1000 biology reports 1: 73 .

39. Gullapalli RR, Desai KV, Santana Santos L, Kant JA, Becich MJ (2012) Next generation sequencing in clinical medicine: Challenges and lessons for pathology and biomedical informatics. Journal of pathology informatics 3: 40 .

40. Diamandis M, White NM, Yousef GM (2010) Personalized medicine: marking a new epoch in cancer patient management. Mol Cancer Res 8(9): 1175-1187.

41. Friend SH, Ideker T (2011) POINT: Are we prepared for the future doctor visit? Nature biotechnology 29: 215.

42. Verhaak RG (2010) Integrated genomic analysis identifies clinically relevant subtypes of glioblastoma characterized by abnormalities in PDGFRA, IDH1, EGFR, and NF1. Cancer cell 17(1): 98-110.

43. Beroukhim R (2010) The landscape of somatic copy-number alteration across human cancers. Nature 463: 899-905.

44. Berger MF (2011) The genomic complexity of primary human prostate cancer. Nature 470: 214-220.

45. Chapman MA (2011) Initial genome sequencing and analysis of multiple myeloma. Nature 471: 467-472.

46. Bass AJ (2011) Genomic sequencing of colorectal adenocarcinomas identifies a recurrent VTI1A-TCF7L2 fusion. Nat Genet 43(10): 964-968.

47. (2011) Network CGAR Integrated genomic analyses of ovarian carcinoma. Nature 474: 609.

48. Timmerman B (2010) Somatic mutation profiles of MSI and MSS colorectal cancer identified by whole exome next generation sequencing and bioinformatics analysis. PloS one 5(12): e15661.

49. Jones SJ (2010) Evolution of an adenocarcinoma in response to selection by targeted kinase inhibitors. Genome biology 11(8): R82.

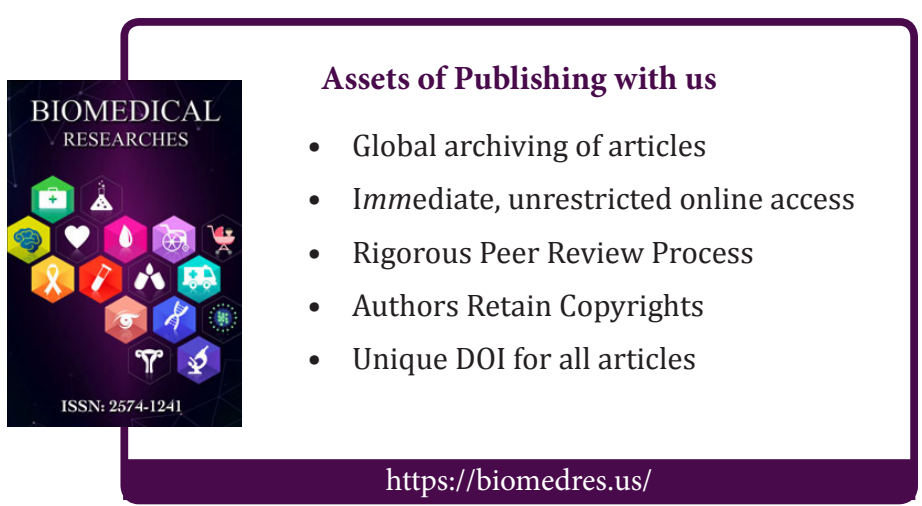

